\title{
Reseña sobre Diseño y desarrollo de tesauros
}

\author{
Viviana Gamba * \\ * Instituto de Investigaciones en Humanidades y Ciencias Sociales (UNLP-CONICET). \\ Facultad de Humanidades y Ciencias de la Educación. Universidad Nacional de La Plata, \\ Argentina | viviana.gamba@gmail.com
}

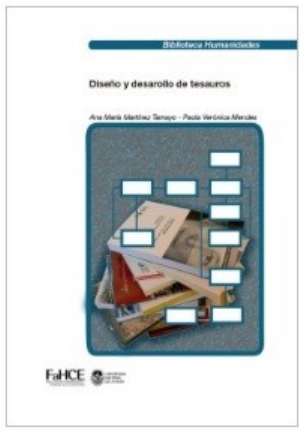

\author{
Reseña de: \\ Diseño y desarrollo de tesauros / Martínez Tamayo, A. M. y Mendes, P. V. (2015). \\ -La Plata: Universidad Nacional de La Plata. Facultad de Humanidades y Ciencias \\ de la Educación. (Biblioteca Humanidades; 38) Recuperado \\ de: http://www.libros.fahce.unlp.edu.ar/index.php/libros/catalog/book/68
}

La obra que se reseña forma parte de la colección Biblioteca Humanidades, editada por la Facultad de Humanidades y Ciencias de la Educación de la Universidad Nacional de La Plata, disponible desde septiembre de 2016 en formato PDF para la descarga libre y gratuita en la categoría Bibliotecología de la página web Libros de la Fahce. Se trata de un manual claro y consistente que ofrece una excelente introducción al tema y aporta información valiosa tanto para estudiantes como para profesionales de la Bibliotecología, la Documentación y la Ciencia de la Información que se enfrentan por primera vez al desafío de diseñar y gestionar un tesauro, o desean actualizar sus conocimientos al respecto.

El tesauro constituye uno de los Sistemas de Organización del Conocimiento (en adelante SOC) más empleados y desarrollados desde mediados del siglo $X X$ y, como tal, ha sido objeto de estudio de numerosos textos especializados y normas de alcance internacional que han pautado su diseño y desarrollo. A través de la presente obra, Martínez Tamayo y Mendes ofrecen un panorama general del tema desde la perspectiva de la Organización del Conocimiento (en adelante $\mathrm{OC}$ ), considerando los avances tecnológicos e incorporando conceptos y pautas actuales, entre otros, los establecidos por la norma ISO 25964-1/2, especialmente enfocada en la publicación electrónica de los tesauros y su interoperabilidad con otros SOC. 
La obra se estructura en doce capítulos a los que se han incorporado, con muy buen criterio, una serie de paratextos preliminares que aportan información práctica para el manejo de la obra identificados con los siguientes títulos: Índice, Etiquetas y símbolos usados en el tesauro, Abreviaturas y Siglas y, finalmente, Prefacio. A ellos se suman los doce cuestionarios de Autoevaluación dispuestos al final de cada capítulo en los que se ofrece una guía práctica para el aprendizaje, que consiste en una serie de preguntas dirigidas al lector y que ponen el acento en aquellos conceptos que, a criterio de las autoras, resultan centrales para lograr una adecuada comprensión de los temas tratados. Por otra parte, se incluyen dos apéndices intercalados en la obra: al final del primer capítulo, el "Apéndice A. Alcance de la Organización del Conocimiento", en el que se aporta la traducción del esquema de clasificación clásico desarrollado por Dahlberg en 1993 y, a continuación del décimo capítulo, el "Apéndice B. Ejemplos de Tesauros Electrónicos", que incluye breves descripciones y capturas de pantalla de varios tesauros disponibles en la web. Finalmente, se ofrece un extenso dispositivo bibliográfico que resulta excelente por su pertinencia respecto a los temas desarrollados, por la inclusión de autores ampliamente reconocidos a nivel internacional y su por actualización al momento de editarse la obra. Al final de la publicación se incluyen un breve apartado titulado "Las Autoras", en el que se brinda una reseña del currículum de Martínez Tamayo y Mendes, y un "Índice de Figuras y Tablas.

En los primeros tres capítulos se presenta el marco conceptual de la obra de modo sintético y claro. En el capítulo 1, se analiza el alcance de la expresión OC a partir de los enunciados expuestos por reconocidos referentes del tema, se mencionan sus orígenes, fundamentos, relaciones con otras disciplinas y controversias respecto al alcance y el uso de las expresiones Organización del Conocimiento, Organización de la Información, Representación del Conocimiento y Gestión del Conocimiento en sus distintos ámbitos de aplicación. A partir de la caracterización del tesauro como un SOC, las autoras ofrecen en el capítulo 2, denominado "Sistemas y Procesos de Organización del Conocimiento", una síntesis acabada de la evolución de estos sistemas, la variación terminológica que se observa para referirse a los mismos conceptos según diversos enfoques disciplinarios y las distintas propuestas tipológicas que permiten clasificar los múltiples SOC en función de diversos parámetros.

A modo de cierre del marco teórico, en el capítulo 3, "Distintos Enfoques para Estudiar los SOC", Martínez Tamayo y Mendes analizan las diversas perspectivas que se aplican al estudio de los SOC en general y los tesauros en particular, los problemas terminológicos implicados y la importancia de aclarar el alcance conceptual de algunas expresiones de origen multidisciplinario usualmente empleadas en el estudio de los SOC. Se analizan los enfoques tradicionales provenientes de la Lingüística Documental y la Terminología y, también, el singular aporte que desde la perspectiva bibliotecológica ofrece del modelo conceptual RFSAD (Functional Requirements for Subject Authority Data), desarrollado por la IFLA.

Una vez establecido el marco teórico general, en el capítulo 4 se define y caracteriza al tesauro como SOC y se describen sintéticamente sus componentes, funciones y tipología. Al respecto, se incluyen consideraciones en relación al empleo de tesauros para la descripción temática en registros bibliográficos de bases de datos convencionales y en esquemas de metadatos. Además, se describen las características de los tesauros de primera generación, en general impresos y poco dinámicos, y los tesauros de segunda generación, creados especialmente en soporte electrónico para ser navegados.

La definición y caracterización del tesauro se complementa en el capítulo 5, "La Historia del Tesauro", con una breve reseña que incluye los principales antecedentes que se inician con la 
explosión de la información de la segunda posguerra mundial, la formulación de la teoría de la indización poscoordinada y el surgimiento de sistemas poscoordinados pioneros como peek-aboo de Batten o Zator de Moores, que las autoras citan y describen de un modo sencillo y comprensible desde la perspectiva de un usuario actual. Luego de mencionar los principales tesauros que marcaron el camino para posteriores desarrollos, las autoras cierran el capítulo con un apartado dedicado a la evolución de las normas y las directrices para el desarrollo de tesauros a nivel internacional, que se complementa con una breve mención a los antecedentes que impulsaron la aparición de los primeros tesauros argentinos y la norma nacional para el desarrollo de tesauros monolingües (IRAM 32057:1983).

Desarrollados hasta aquí todos los aspectos que definen el contexto del tema central de la obra, Martínez y Mendes destinan los siguientes apartados al tratamiento específico del diseño y el desarrollo de tesauros como un proceso complejo que puede abordarse desde la perspectiva de la gestión de proyectos. Al inicio del capítulo 6, las autoras proponen a sus lectores reflexionar sobre las posibilidades y las necesidades reales de desarrollar un tesauro en todos los casos que parecieran requerir esta tarea. Para guiar esta reflexión ofrecen una serie de consignas simples y prácticas que los lectores podrán formularse antes de planificar un nuevo tesauro. Tomada la decisión de avanzar con el proyecto, se propone un recorrido por los aspectos centrales a tener en cuenta, como la conformación de un equipo de trabajo, la formulación de un plan de acción, la provisión de recursos financieros, físicos, bibliográficos, normas, manuales y programas informáticos para la gestión de tesauros. Siguiendo con el capítulo 6, en el apartado "Hacer el tesauro", se describen brevemente los métodos que pueden emplearse: deductivo (teórico, sintético, a priori o top-down), inductivo (empírico, analítico, a posteriori o bottom-up), mixto o híbrido. A continuación, se examinan la serie de procesos de OC que permiten el diseño y el desarrollo de tesauros: Definición y análisis del dominio, Control de vocabulario, Determinación de relaciones jerárquicas y asociativas, Construcción del orden sistemático y Publicación del tesauro. Esto pasos se complementan con la evaluación del tesauro y su mejora continua. En el cierre del capítulo, y teniendo en cuenta que "un tesauro no surge de la nada" (Martínez y Mendes, 2015, p. 96), se ofrecen consideraciones precisas en relación al principio de garantía que asegura el respaldo intelectual del contenido del tesauro.

En los capítulos 7 y 8 se desarrollan dos aspectos fundamentales e inherentes a la conformación de un tesauro. Ellos son: vocabularios de indización y de entrada y relaciones jerárquicas y asociativas. A continuación, en el capítulo 9, "El Orden Sistemático del Tesauro", se ofrece información para la construcción de un esquema o plan de organización de los conceptos correspondientes al dominio del tesauro incorporando los principios del análisis de facetas, los tipos de orden sistemático aceptados por la norma ISO 25964 y otros conceptos relacionados con la notación. Finalmente, en el capítulo 10, "Evaluación, Publicación y Actualización del tesauro", se exponen los métodos a emplear y los momentos en los que se deben llevar adelante las evaluaciones. Por otra parte, se brindan consideraciones respecto a la edición y la publicación del tesauro, teniendo en cuenta las diferencias entre los tradicionales impresos y los desarrollados en soporte electrónico incluyendo, además, los requerimientos de actualización para ambos casos.

A modo de cierre, en el capítulo 11, "El Tesauro y la Interoperabilidad", las autoras ofrecen una introducción al tema de la interoperabilidad estableciendo su alcance conceptual, describiendo los modelos de interoperabilidad entre SOC establecidos en la norma ISO 25964-2, los tipos de mapeo que permiten determinar dicha interoperabilidad y una serie de problemas frecuentemente detectados en tesauros multilingües. El capítulo concluye con la presentación 
breve de algunas experiencias de interoperabilidad conocidas a nivel internacional realizadas entre varios tesauros o entre tesauros y otros SOC.

En síntesis, Diseño y Desarrollo de Tesauros constituye un aporte significativo a la bibliografía en español disponible sobre el tema, una auténtica guía para la formación, actualización y práctica profesional aplicada al desarrollo de tesauros que logra con creces el objetivo que las autoras se propusieron en el Prólogo, al señalar que deseaban ofrecer una "herramienta útil para aquellas personas a quienes está dirigida" (Martínez Tamayo y Mendes, 2015, p.11). 